\title{
EXTRACOELOMIC APPROACH TO THE SPINE
}

\author{
RONALD MOSKOVICH, DANIEL BENSON, ZHI-HU ZHANG, MARK KABINS
}

From the Hospital for Joint Diseases, New York, and the University of California Davis, Sacramento, USA

\begin{abstract}
A modified transthoracic approach to the thoracic vertebral column is described. In this method, the parietal pleura is detached from the chest wall and retracted with the visceral pleura and its contents. A direct approach to the vertebral bodies is thus achieved without transgression of the intrapleural space. The technique can be extended to include exposure of the thoracolumbar spinal column, utilising a thoracoabdominal approach with extrapleural and extraperitoneal dissection. Management of the costophrenic detachment is thus simplified. This approach has significant advantages for orthopaedic, vascular and neurosurgical procedures.
\end{abstract}

J Bone Joint Surg [ Br] 1993; 75-B :886-93.

Received 15 February 1993; Accepted 29 March 1993

The anterior surgical approach to the thoracic and lumbar spine was popularised by Hodgson and Stock (1957), who reported the technique in a short paper and later published an extensive series (Hodgson et al 1960). A combination of extrapleural and transpleural techniques was used. An extraperitoneal approach to the spine had been previously described as a means of reaching the sympathetic chain to perform lumbar sympathectomy (Royle 1924). Pearl (1937) described a retroperitoneal approach to the lumbar sympathetic chain via a muscle-splitting approach. Several surgeons have recommended variations of these techniques (Flothow 1935, 1951; Pratt 1950) but the definitive descriptions of the surgical approach were given by Lilly et al (1954) and by Southwick and Robinson (1957) who described its use for spinal surgery.

Harmon (1948) reported his experience with anterior lumbar discectomy. Anterior exposure of the thoracolumbar spine has traditionally used the transthoracicretroperitoneal approach (Burrington et al 1976; McAfee and Zdeblick 1989) while the standard anterior approach

R. Moskovich, MD, FRCS, Assistant Professor of Orthopaedic Surgery Z.-H. Zhang, MD, Research Fellow

Hospital for Joint Diseases Orthopaedic Institute, 301 East 17th Street, New York, NY 10003, USA.

D. Benson, MD, Professor of Orthopaedic Surgery

M. Kabins, MD, Spine Fellow

University of California, Davis, 2230 Stockton Boulevard, Sacramento,

CA 95817, USA.

Correspondence should be sent to Dr R. Moskovich

(C)1993 British Editorial Society of Bone and Joint Surgery $0301-620 \mathrm{X} / 93 / 6644 \$ 2.00$ to the thoracic spine is transthoracic. Thoracotomy is performed, the parietal pleura and periosteum are incised deep to a rib, and the pleural cavity is entered. The lungs (and mediastinum) which are enveloped by the visceral pleura are retracted, and the posterior (costovertebral) parietal pleura is incised longitudinally to expose the underlying vertebral column. The parietal pleura has then to be repaired, which may increase the risk of intrapleural adhesions.

Costotransversectomy provides a safe extrapleural approach to the spine for vertebral biopsy, abscess drainage and the treatment of short-segment spinal instability or neuraxial compression (Capener 1954). The exposure is not really extensile and remains limited although multiple rib resection gives additional exposure (Hyndman 1947; Fessler et al 1991).

Mirbaha (1973) described an extrapleural-retroperitoneal approach to the thoracolumbar junction. The exposure was limited to resection of the twelfth rib, with a complex closure of the incision. An earlier report by Fey (1925) had described an extrapleural-extraperitoneal approach and Francioli (1951) subsequently clearly described and illustrated a combined extrapleural and retroperitoneal approach to the thoracolumbar and lumbar sympathetic chain. In his technique the dissection is performed both above and below the diaphragm, maintaining the costophrenic attachment, and stripping the pleura and peritoneum off the superior and inferior surfaces of that muscle. Although this approach may be adequate for biopsy or surgery on the sympathetic chain, retention of the diaphragmatic attachment limits exposure of the spinal thoracolumbar junction.

We now describe an extensile extracoelomic (extrapleural and extraperitoneal) approach to the thoracic and lumbar spine. It simplifies the management of the diaphragm and may reduce the incidence of complications of the transpleural approach such as implants not being covered by parietal pleura, intrapleural migration of bone grafts, and adhesions within the pleural cavity.

\section{SURGICAL TECHNIQUE}

Standard anaesthetic techniques, as for thoracotomy, are required. The use of a double-lumen (Carlen) endotracheal tube to allow selective inflation or deflation of the lungs is generally not necessary. Intubation proximal to the carina is usually sufficient.

The patient is positioned in the full lateral position 


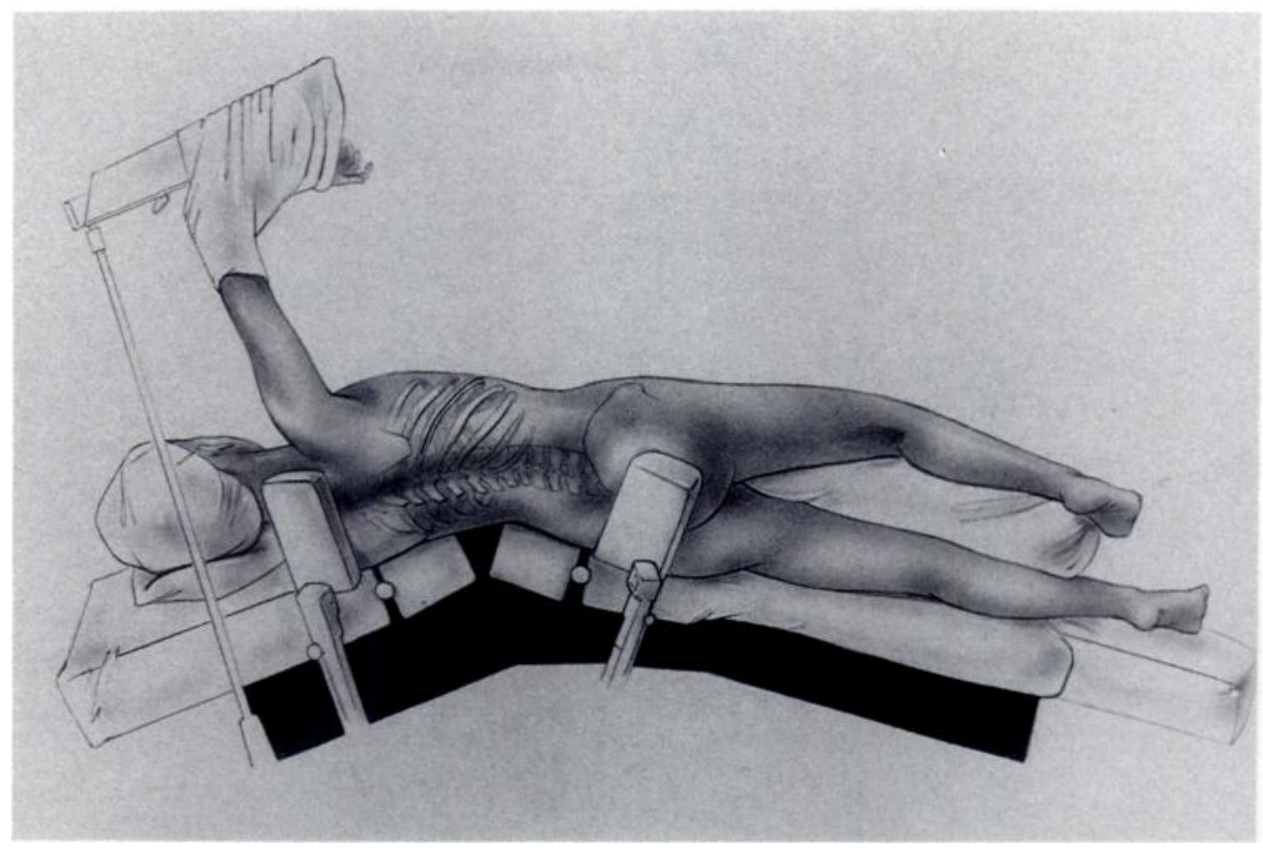

Fig. 1

Position of the patient on the operating table. A roll is necessary beneath the lower axilla to protect the brachial plexus from pressure. The incision (overlying the eighth rib in this illustration) is indicated by the dark line. Elevation of the central kidney supports aids the exposure. They should be lowered during closure of the incision.

with kidney rests supporting the pelvis and shoulders posteriorly and the operating table tilted backwards approximately $30^{\circ}$ (Fig. 1), thus firmly immobilising the patient by gravity. A small roll under the dependent axilla avoids compression of the axillary neurovascular bundle. The superior arm should be supported on a pillow or suspended from a bar attached to the operating table. Abduction of the arm elevates the scapula, optimises access to the proximal ribs, and also allows adjustment of the table height without altering the arm position. The operating table may also be arched or 'broken' or the kidney rests elevated, to draw the lower ribs away from the iliac crest, to elongate the lateral aspect of the thorax and to assist in enlarging the entry portal into the chest cavity. Flexion of the hip and knee on the same side as the incision further aids exposure by relaxing the psoas muscle.

The extrapleural thoracotomy. In the modified extrapleural approach the potential intrapleural space remains inviolate, and wide spinal exposure is obtained. The skin incision varies with the spinal level and ribs which are to be exposed. It should be at least $2 \mathrm{~cm}$ distal to the tip of the scapula even if the third or fourth ribs are involved, in which case the scapula is retracted with a large blunt rake or scapular retractor. The incision extends from the posterior angle of the rib to the costochondral junction and is carried down through the subcutaneous tissues to the muscles, which are identified and incised by electrocautery. The latissimus dorsi and the serratus anterior muscles are divided in the line of the skin incision, and the ribs are exposed (Fig. 2A). It is not necessary to incise the erector spinae muscles posteriorly as this does not improve the exposure and will only increase the blood loss. The rib can be exposed quite adequately deep to the paraspinal muscles.

Either intercostal or transcostal entry into the thoracic cavity is possible. The periosteum over the selected rib is incised and elevated using Alexander, Matsen and Doyen periosteal elevators so that the rib is completely exposed. The chest cavity can then be entered with or without resection of the rib (Fig. 2B). The periosteum in the rib bed is incised, with care taken not to injure the parietal pleura which is loosely adherent to the periosteum (Fig. 2C). A plane can usually be developed between the two, however, using a small blunt dissector such as a dental roll on a haemostat (Fig. 2D). The parietal pleura is progressively separated from the chest wall, working posteriorly, proximally, and distally towards the vertebral column, the cupula, and the diaphragm respectively. Blunt dissection is by a spongecovered finger or a sponge-stick. When the superior surface of the diaphragm is exposed, the parietal pleura with its enclosed visceral pleura and viscera are retracted medially. The vertebral column with the overlying segmental vessels, the sympathetic chain and the thoracic duct, the ribs and the subcostal neurovascular bundles are thus exposed (Fig. 3).

Exposure through the bed of a single rib will usually allow the performance of discectomy at several levels. The approach through the fifth rib is useful unless the lowest thoracic vertebrae are to be reached, in which case it is better to go through the seventh or eighth ribs. 


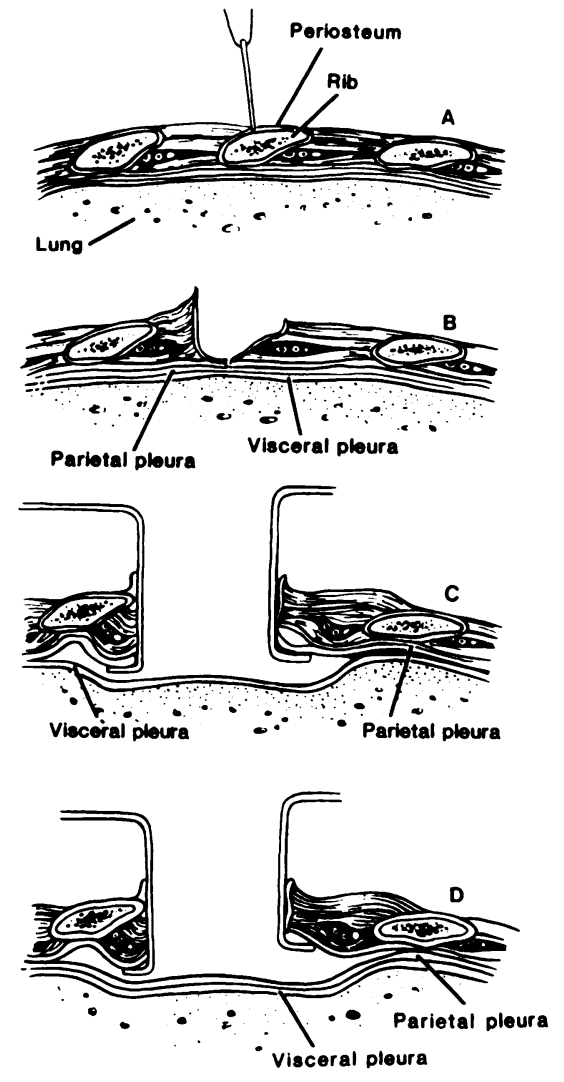

Fig. 2

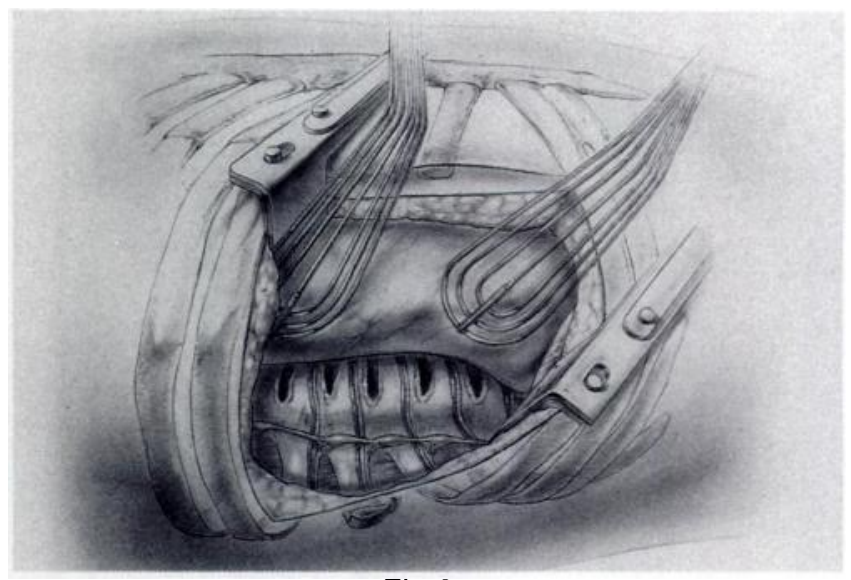

Fig. 3

Figure 2 - Initial dissection is performed down to the rib, and the periosteum is incised along its midline (A). The rib is exposed subperiosteally and removed. It is retained for use as bone graft (B). The plane of entry into the chest cavity for the transpleural approach is directly through the parietal pleura, into the intrapleural space (C). The plane of dissection for the extrapleural approach is external to the parietal pleura. The parietal pleura should be carefully dissected off the chest wall (D). (Illustrations modified from: Hoppenfeld S and de Boer P. Surgical exposures in orthopaedics. Philadelphia: J.B. Lippincott, 1984.). Figure 3 - Extrapleural exposure of the right hemithorax. The parietal pleura and the enclosed visceral pleura and lung are retracted medially. The superior surface of the diaphragm can be seen through the lower part of the incision. The intercostal vessels and the sympathetic chain can be clearly visualised after the overlying pleura has been reflected. Discectomy can be performed without injury to the segmental vessels.
The fifth rib is usually deep to the scapula in its normal position and an incision at this level seldom results in discomfort or clicking as the scapula glides over the thoracic cage. The rib level selected should generally correspond to the lowest disc to be exposed. Individual variations in the horizontality of the ribs, the flexibility of the rib cage and the magnitude of any spinal deformity also affect access.

It may be convenient to perform an intrathoracic costoplasty if there is a severe scoliosis and deformity of the rib cage. The extrapleural exposure of the thoracic wall facilitates this procedure as all the ribs are visible with no additional incision through the parietal pleura.

The 'double' thoracotomy. If anterior thoracic instrumentation is to be inserted then the approach may be made through two rib beds, for instance the fourth and the eighth, incising the periosteum at both levels. The skin incision should be more oblique than for single rib access. If this double approach is used then we recommend that the ribs are not excised, although some bone may be obtained for grafting by splitting them longitudinally and resecting only one part.

Closure and wound drainage. It is always necessary to drain the surgical site. A chest tube connected to an underwater seal is placed in the extrapleural space and tunnelled distally to emerge in a distal intercostal space in the midaxillary line. The intercostal tube runs obliquely through the chest wall so that it is not angulated when the patient lies supine; drainage is facilitated by gravity.

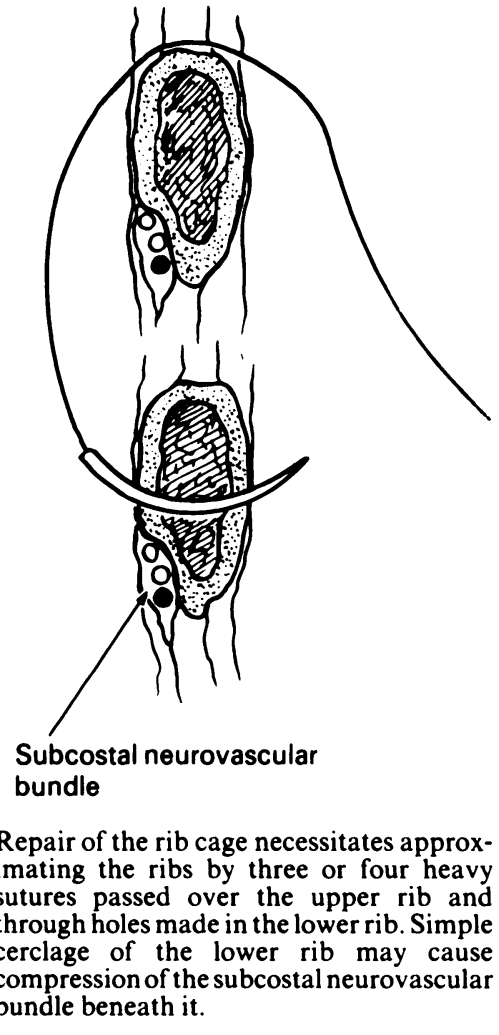

After removal of the tube, an adhesive dressing prevents entry of air from outside. If the parietal pleura has been accidentally torn it may be repaired or left open. A 
second chest tube is usually unnecessary but should the patient develop a postoperative pleural effusion, an additional drain can be inserted into the intrapleural space.

The wound is closed by first repairing the periosteal bed of the rib with interrupted, size 0 Vicryl sutures. It is sometimes helpful to approximate adjacent ribs by a double-armed size 1 nylon cerclage suture. This should pass proximal to the upper rib, and through a hole made in the lower rib so that the lower subcostal neurovascular bundle is not compressed (Fig. 4). Further repair is then done as for a standard thoracotomy by repairing the chest wall in layers.

Postoperative care includes maintenance of intercostal drainage until there is less than $40 \mathrm{ml}$ discharged in 8 to 12 hours. The volume drained varies with the size of the exposure, the extent of the fusion and whether or not an intrathoracic costoplasty has been performed.

Thoracoabdominal exposures. Thoracoabdominal exposures can also be performed by the extrapleural route (Fig. 5). We generally use the ninth or tenth rib bed, which is more distal than for the thoracic approach. The incision crosses the costochondral junction and passes obliquely across the abdominal wall towards the lateral border of the rectus abdominis muscle, not violating the lateral edge of the rectus sheath. Alternatively, it may be more vertical, extending down to a point 2 to $3 \mathrm{~cm}$ superomedial to the superior iliac crest. The thoracic exposure is performed as described above. The rib is disarticulated at its costochondral junction, and the costal cartilage transected to initiate the abdominal exposure. This provides a cartilaginous landmark for use during closure. The external oblique muscle is usually split in the line of its fibres, as they run from superolateral to inferomedial, or it may be necessary to incise the muscle by electrocautery. The underlying internal oblique muscle is then divided, followed by the transversus abdominis muscle. Once the fascia of the transversus abdominis has been incised, fat becomes visible in the extraperitoneal space. A plane is then developed by fingertip dissection between the peritoneum and the posterior abdominal wall. The ureter usually adheres to the parietal peritoneum and is elevated off the psoas muscle during the dissection. A common mistake is to reflect the retroperitoneal fat with the peritoneum; this leads to a blind space posterior to the psoas muscle. Instead, the fatty layer should be separated from the peritoneum to create a plane leading down to the anterior aspect of the psoas and the vertebral bodies (Southwick and Robinson 1957). Blunt dissection of the retroperitoneal tissues proximally towards the undersurface of the diaphragm exposes the costophrenic insertion, the peripheral attachment of the diaphragm to the abdominal wall. The surgeon's fingers should meet on either side of the diaphragm at the costophrenic margin. Confluence of the extrapleural and the retroperitoneal spaces is achieved by detaching the diaphragm from its insertion on the chest wall and the crus from the side of the spine. It is not necessary to expose much of the diaphragmatic muscle, but rather it is left sandwiched between the pleura and the peritoneum (Fig. 6).

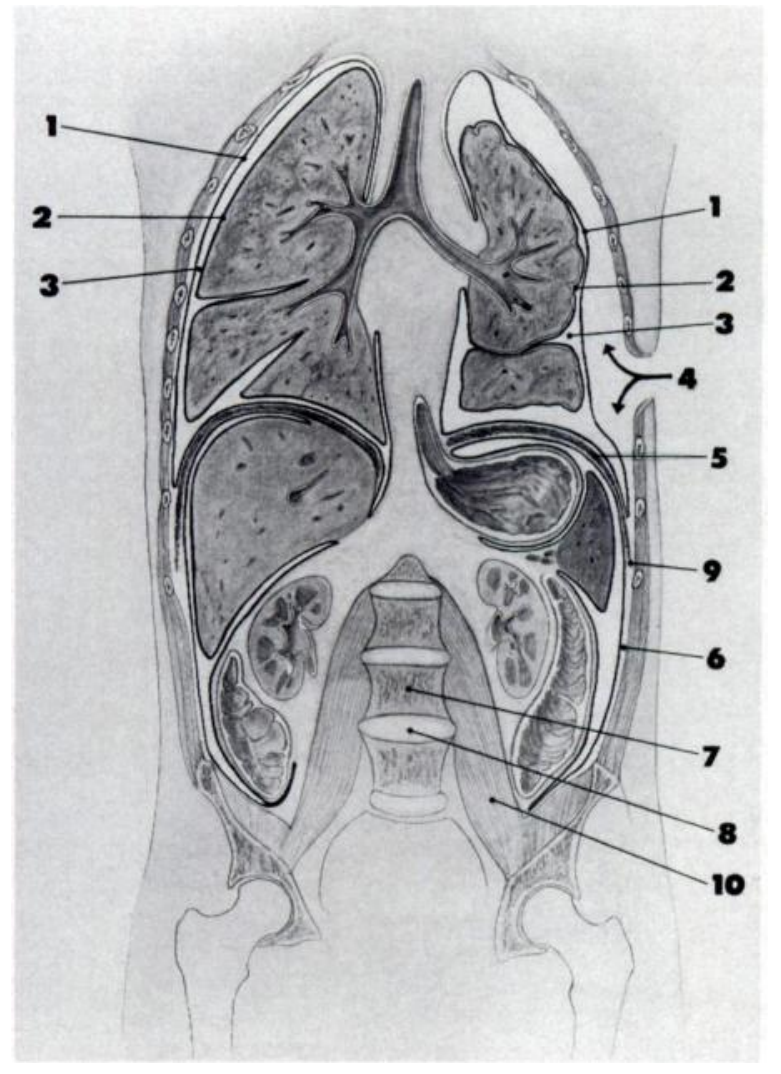

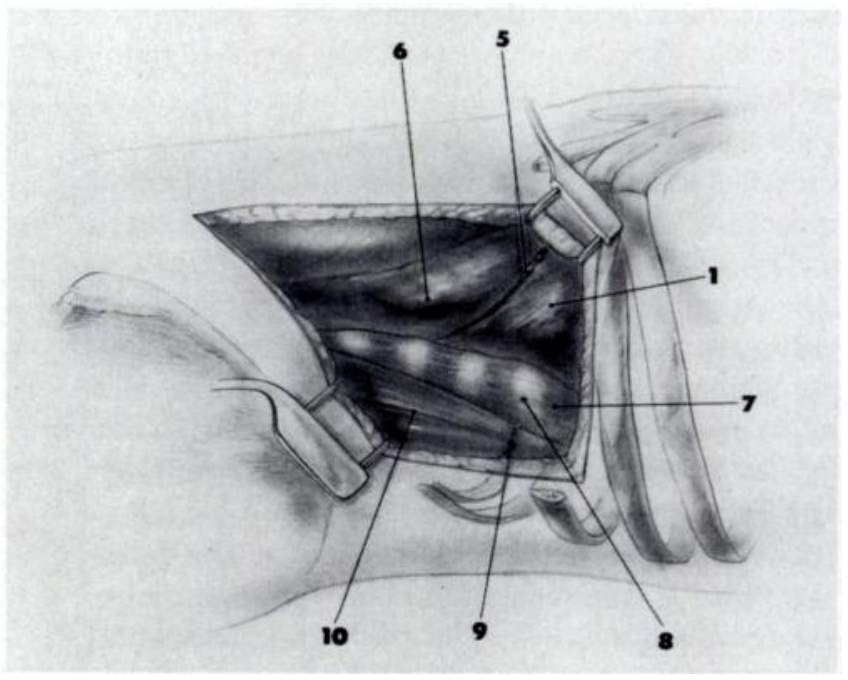

Fig. 5

Fig. 6

Figure 5 - Diagrammatic representation of the extracoelomic approach to the spine seen in coronal section of the trunk. Figure 6 - Left thoracoabdominal exposure. The patient is lying on the right side. The thoracic and lumbar vertebrae can be seen. The cut edge of the diaphragmatic muscle is shown, sandwiched between the pleura above and the peritoneum below. 1, parietal pleura; 2 , visceral pleura; 3 , pleural cavity; 4, extrapleural space; 5 , diaphragm; 6 , peritoneum; 7 , vertebra; 8 , intervertebral disc; 9 , costophrenic junction; 10 , psoas major. 
After completion of the spinal operation, the entire pleural/peritoneal 'tube' is allowed to return to its normal position and a large intercostal chest drain is laid in the extrapleural-retroperitoneal space if necessary. No attempt is made to reattach the diaphragm to the chest wall; closure is by repair of the chest wall in layers. Approximation of the costal cartilage facilitates correct alignment of the tissue plane during the repair. Rapid readhesion of the entire chest and abdominal wall occurs with restoration of normal diaphragmatic and pulmonary function.

\section{CLINICAL STUDY}

Methods. The records of 65 consecutive patients who had undergone thoracotomy or a thoracoabdominal approach over a two-year period were examined. Thirty-eight were operated upon using the extracoelomic approach and 27 had a transthoracic or transthoracic-retropleural approach. Preoperative, surgical and postoperative data were available for all the patients and all were followed up for at least two years. Statistical analysis was performed using the chi-squared test and Student's $t$-test to assess the null hypothesis that there were no significant differences between the transpleural and the extrapleural operations. Analysis of variance (ANOVA) was performed and the data were also examined using the MannWhitney $\mathrm{U}$, the Wilcoxon rank-sum $\mathrm{W}$, and the Kolmogorov-Smirnov two-sample tests to determine whether the groups came from similar populations and had similar distributions.

Results. The mean age of the patients was $24.2 \pm 15.6$ years ( 3 to 75$)$. There was no significant age difference between the two groups $(p=0.4)$. The anterior approach to the spinal column had been most commonly used for the correction of spinal deformity. Fifty-three patients had undergone anterior release and arthrodesis for scoliosis. In 30 it was idiopathic, in 12 due to cerebral palsy, in 9 to other neuromuscular disorders, in one to neurofibromatosis, and in one it was congenital (Fig. 7). The approach had also been used for vertebrectomy and for anterior debridement and fusion (for infection in two patients, for correction of kyphosis in three and for ankylosing spondylitis in one). Six patients who had burst fractures with significant narrowing of the spinal canal had also been operated on using the anterior approach. Non-ambulators formed $32 \%$ of the patients, mainly those with cerebral palsy. The other patients, except for those with burst fractures, were all neurologically normal.

Some details of the 38 thoracotomies and 27 thoracoabdominal approaches are given in Table I. Entry into the chest cavity was effected through the rib beds of the fourth to the twelfth ribs. Thoracotomy was generally performed through the fifth to eighth ribs and thoracoabdominal approaches via the lower ribs (Fig. 8). In the patients with deformity, the mean number of interverte- bral disc levels fused was $5.1 \pm 1.4$ (Fig. 9), and Zielke instrumentation (or Dwyer in one patient) was used in $39 \%$ of these spines. In trauma patients the mean number of motion segments fused anteriorly was 2.5 and in patients with vertebral infections it was one level. No internal fixation was used in the patients without deformity. No significant difference was seen in the number of levels involved in the two techniques $(\mathrm{p}=$ $0.4, \mathrm{df}=7$ ).

The mean intraoperative blood loss for the whole group was $925 \pm 1591 \mathrm{ml}(100 \mathrm{ml}$ to $10 \mathrm{l})$. Three patients who had undergone the extrapleural approach had intraoperative blood loss greater than twice the standard deviation from the mean. In these it was thought not to be directly related to the surgical approach used; two of them had complex adult scoliosis and one had a kyphosis due to ankylosing spondylitis (Fig. 10). No patient in the other group had undergone procedures of equivalent

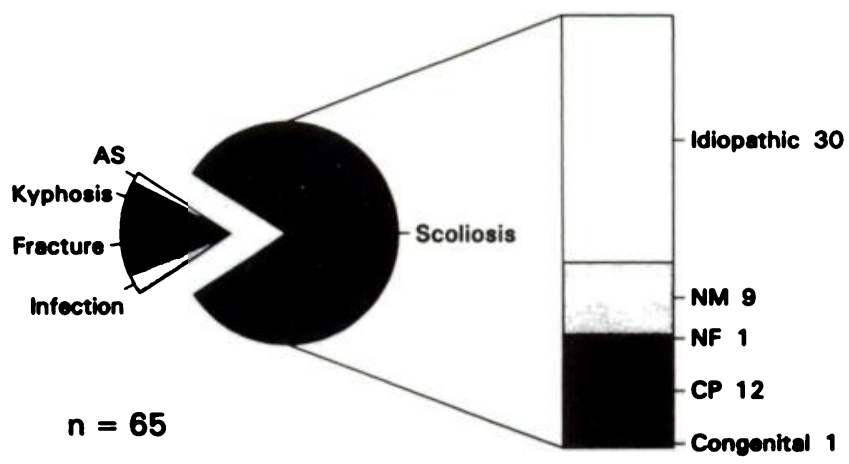

Fig. 7

Diagnoses of the patients who underwent anterior thoracic or thoracoabdominal surgery. $\mathrm{AS}=$ ankylosing spondylitis: $\mathrm{NM}=$ neuromuscular scoliosis: $\mathrm{NF}=$ neurofibromatosis; $\mathrm{CP}=$ cerebral palsy.

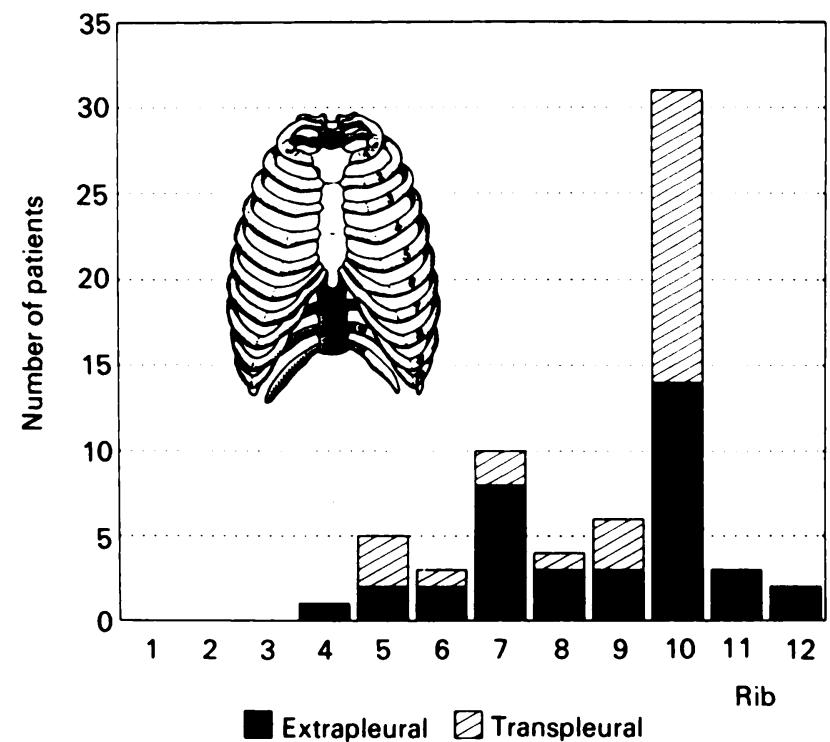

Fig. 8

The rib levels used for the anterior approach. 


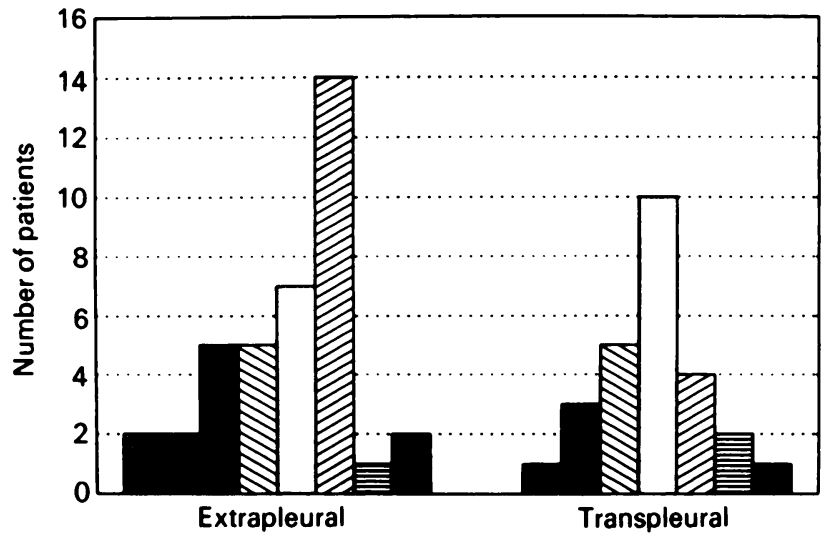

$1 \square 2 \square 3 \mathrm{~B}_{4} \square 5 \square_{6}$ 晨 $7 \square_{8}$

Number of motion segments fused

Fig. 9

The number of motion segments arthrodesed per patient. The difference between the groups was not statistically significant.

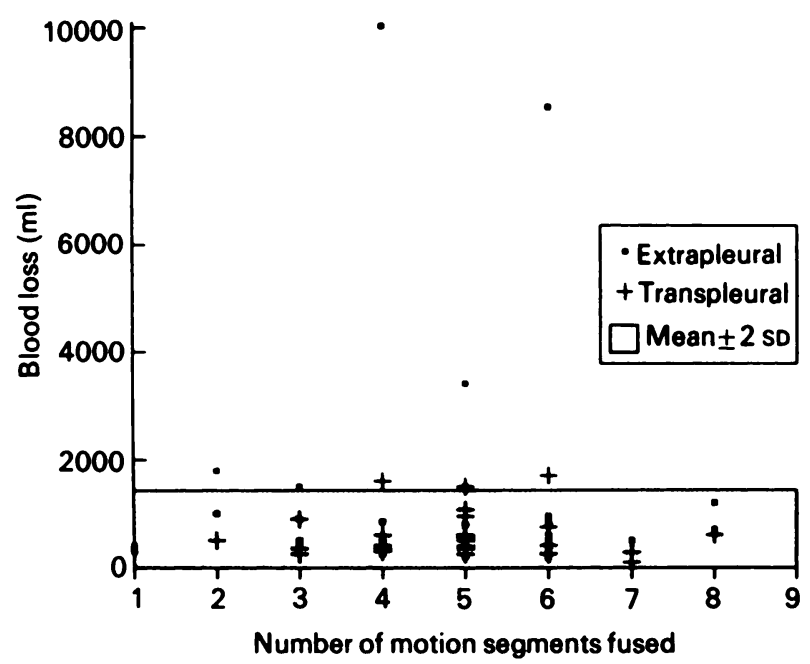

Fig. 10

The operative blood loss for the two groups of patients. The mean surgical blood loss was $616 \pm 409 \mathrm{ml}$ if the three outlying values are excluded from the calculation. The median and the mode for the entire group was $500 \mathrm{ml}$.

severity and complexity and these three patients were therefore excluded from the comparison of operative blood loss. There was then no significant difference in blood loss between the two surgical approaches $(p=$ $0.9)$.

There was no significant difference in the use of chest tubes between the extrapleural and transpleural approaches $(p=0.07, d f=2)$. Six patients who had an extrapleural exposure of the spine also had an intrathoracic costoplasty; none of the transpleural patients was so treated. Pleural effusion occurred in $31 \%$ of all patients and was significantly more frequent in the extrapleural group ( $\mathrm{p}=0.003)$. There was no significant relationship between the development of an effusion and the use of
Table I. Statistical comparison of the extrapleural versus the transpleural approach to the thoracic and thoracolumbar spine

\begin{tabular}{lccl}
\hline & $\begin{array}{l}\text { Extrapleural } \\
(\mathbf{n}=\mathbf{3 8})\end{array}$ & $\begin{array}{l}\text { Transpleural } \\
(\mathbf{n}=\mathbf{2 7})\end{array}$ & p value \\
\hline Thoracoabdominal & 19 & 8 & 0.1 \\
Thoracotomy & 19 & 19 & \\
$\begin{array}{l}\text { Side } \\
\quad \text { Left } \\
\text { Right }\end{array}$ & 19 & 12 & 0.7 \\
$\quad$ Ambulatory & 19 & 15 & \\
Morbidity & 23 & 21 & 0.1 \\
Internal fixation & 6 & 5 & 0.8 \\
Posterior fusion & 11 & 11 & 0.3 \\
Chest tube & 27 & 20 & 0.8 \\
$\quad$ 2 & & & \\
Effusion & 2 & 27 & 0.07 \\
Chest tube output (ml) & $628 \pm 374$ & $948 \pm 601$ & 0.1 \\
Age (years) & $22.8 \pm 16.0$ & $26.1 \pm 15.2$ & 0.4 \\
Length of stay (days) & $23.9 \pm 12.6$ & $19.6 \pm 11.9$ & 0.2 \\
Operative time (min) & $315 \pm 118$ & $287 \pm 95$ & 0.3 \\
Surgical blood loss (ml) & $624 \pm 402 *$ & $605 \pm 426$ & 0.9 \\
$\begin{array}{l}\text { Number of segments fused } \\
\text { per level } \dagger\end{array}$ & $4.8 \pm 1.7$ & $4.9 \pm 1.4$ & 0.9 \\
\hline
\end{tabular}

* three patients excluded from calculation (see text) †see Figure 9

chest tubes $(p=0.3)$ or the number of levels operated on.

All non-respiratory postoperative complications were considered in the 'morbidity' evaluation (Table I). No significant difference was found between the two groups $(p=0.8)$. Most of the complications were minor such as postoperative urinary tract infection, and all resolved without sequelae.

One patient developed atelectasis that resolved after fibreoptic bronchoscopy and removal of a mucus plug. Three patients required insertion of an additional intercostal tube for the drainage of persistent pleural effusions. One death occurred 12 days postoperatively in a patient with cerebral palsy who had undergone both anterior and posterior fusion. He developed glottic oedema and died before an adequate airway could be established. None of the patients who had the diaphragm detached developed problems of diaphragmatic dysfunction. Four patients who had undergone thoracoabdominal surgery were evaluated fluoroscopically at follow-up examination and they all had normal diaphragmatic motion with respiration. There was no evidence of paradoxical movement.

Statistical analysis of the data for the extracoelomic and the transpleural groups confirmed the null hypothesis for the two types of surgical exposure for laterality of incision, use of anterior spinal instrumentation, posterior 
arthrodesis, placement of intercostal tubes and chest tube output, patient's age, length of hospital stay, operative time, surgical blood loss, postoperative complications and the number of motion segments arthrodesed. Patients who had undergone an extrapleural approach were, however, significantly more likely to have an effusion.

\section{DISCUSSION}

The unique features of the extrapleural approach to the spine are that a potential extrapleural space exists and that surgical repair of the costophrenic junction is simplified.

The embryological development of the pleural and peritoneal cavities provides the basis for the extracoelomic surgical approach both to the thoracic and to the thoracolumbar spine. By the middle of the second week of intrauterine life, small spaces lined by mesothelial cells appear on each side of the embryo in the lateral plate mesoderm. These spaces become confluent and form the intraembryonic coelom or body cavity which is lined by the intraembryonic parietal, or somatopleuric mesoderm in contact with the ectoderm, and the intraembryonic visceral, or splanchnopleuric mesoderm in contact with the endoderm. The endothelial heart tubes then invaginate the cavity, forming the pericardium and creating two lateral pericardio-peritoneal canals. These are then invaginated by the lung buds at about 32 to 33 days and become the pleural cavities. Thus, at this stage the intraembryonic coelom consists of a median unpaired pericardial cavity, two primitive pleural cavities and the unpaired peritoneal cavity (Hamilton 1976a). A crescentic ridge of mesoderm then projects into the most caudal part of the pericardio-peritoneal canal. Eventually the membrane extends medially to close the communication between the pleural and peritoneal cavities thus forming part of the diaphragm. Muscle appears in the pleuroperitoneal membrane with myoblasts derived from the third, fourth and fifth cervical somites (Hamilton 1976b).

The muscular diaphragm is thus sandwiched between the parietal pleura and the peritoneum, and may be detached from the trunk wall without serious concern about either caudal or rostral displacement of the attachment. A single chest tube placed in the extrapleuralretroperitoneal space encourages the membranes to

\section{REFERENCES}

Burrington JD, Brown C, Wayne ER, Odom J. Anterior approach to the thoracolumbar spine: technical considerations. Arch Surg 1976; $111: 456-63$.

Capener N. The evolution of lateral rhachotomy. J Bone Joint Surg [Br] 1954; 36-B:173-9.

Dommisse GF. The blood supply of the spinal cord: a critical vascular zone in spinal surgery. J Bone Joint Surg [Br] 1974; 56-B:225-35.

Enneking WF. Musculoskeletal tumor surgery. Vol. 1. New York: Churchill Livingstone, 1983;90. readhere to the trunk wall. Concomitant intrapleural placement of a chest tube of small diameter may reduce the incidence of postoperative pleural effusion.

The side exposed depends mainly on the reasons for the operation, and on the pathological anatomy of the spine. If scoliosis is greater than $30^{\circ}$, the great vessels lie within the concavity of the deformity and interfere with exposure of the spine from that side; entry into the thoracic cavity should be from the opposite side. If the pathology does not necessitate a particular exposure, then it should be borne in mind that it is easier to mobilise the aorta off the spine than the vena cava. The latter, however, is a low-pressure vessel, and less likely to be injured by adjacent implants. Right-sided thoracic exposures have the general advantage of not requiring retraction or mobilisation of the aorta and the heart.

Resection of malignant spinal tumours should probably be performed via transpleural thoracotomy so that the tumour can be removed en bloc with the overlying parietal pleura. Only in this manner can a 'wide' margin, as defined by the Musculoskeletal Tumor Society (Enneking 1983) be obtained. This principle should also apply to lumbar spinal tumour resections although most approaches to tumours in this area are extraperitoneal rather than transabdominal.

It is now possible to perform discectomy, vertebrectomy, vascularised rib transfers, and spinal instrumentation without transgressing the pleural cavity. It is prudent, however, to avoid anterior spinal implants which are prominent or have sharp edges or points (Wong, Waters and Walesby 1991) because of the risk of visceral or vascular injury.

We think that multilevel discectomies should be done without ligating the segmental vessels. This is easier to achieve with the wider exposure afforded by the extrapleural approach. Avoidance of vessel ligation reduces the risk of ischaemic damage to the spinal cord by inadvertent interference with the artery of Adamkiewicz or damage to the segmental blood supply in the vascular watershed region of the spinal cord (Dommisse 1974). If, however, the vertebral bodies are instrumented, then the vessels should be ligated to prevent haemorrhage.

No benefits in any form have been received or will be received from a commercial party related directly or indirectly to the subject of this article.
Fessler RG, Dietze DD, MacMillan M, Peace D. Lateral parascapular extrapleural approach to the upper thoracic spine. J Neurosurg $1991 ; 75: 349-55$.

Fey B. L'abord du rein voie thoraco-abdominale. Urologicale Clin Necker 1925; 5:169-78.

Flothow PG. Anterior extraperitoneal approach to the lumbar sympathetic nerves. Am J Surg 1935; 29:23-5.

Flothow PG. Observations on twenty years of experience in surgery of the sympathetic nervous system. West J Surg 1951; 59:110-6. 
Francioli P. Voies d'accès dans les sympathectomies lombaire et lombothoracique. Helv Chir Acta 1951; 18:536-56.

Hamilton WJ, Mossman HW. Hamilton Boyd and Mossman's human embryology. Fourth ed. Baltimore, etc: Williams and Wilkins, 1976a; IV : 78-80.

Hamilton WJ, Mossman HW. Hamilton Boyd and Mossman's human embryology. Fourth ed. Baltimore, etc: Williams and Wilkins, 1976b; XI:367-73.

Harmon PH. The removal of lower lumbar intervertebral discs by the transabdominal extraperitoneal route: a preliminary report of three cases. Permanente Foundation Medical Bulletin 1948; 6: 169-74.

Hodgson AR, Stock FS. Anterior spinal fusion: a preliminary communication on the radical treatment of Pott's disease and Pott's paraplegia. Br J Surg 1956-1957; 44:266-75.

Hodgson AR, Stock FE, Fang HSY, Ong GB. Anterior spinal fusion: the operative approach and pathological findings in 412 patients with Pott's disease of the spine. Br J Surg 1960-1961; 48:172-8.

Hyndman OR. Transplantation of the spinal cord: the problem of kyphoscoliosis with cord signs. Surg Gynecol Obstet 1947; 84: 460-4.
Lilly GD, Smith DW, Biggane CF, Jana JT. An evaluation of 'high' lumbar sympathectomy in arteriosclerotic circulatory insufficiency of the lower extremities. Surgery $1954 ; 35: 1-8$.

McAfee PC, Zdeblick TA. Tumors of the thoracic and lumbar spine: surgical treatment via the anterior approach. J Spinal Disord 1989; 2:145-54.

Mirbaha MM. Anterior approach to the thoraco-lumbar junction of the spine by a retroperitoneal-extrapleural technic. Clin Orth 1973; $91: 41-7$.

Pearl FL. Muscle-splitting extraperitoneal lumbar ganglionectomy. Surg Gynec Obstet 1937; 65:107-12.

Pratt GH. The present status of sympathectomy in the treatment of vascular diseases. Angiology 1950; 1:9-19.

Royle ND. The treatment of spastic paralysis by sympathetic ramisection: experimental basis and clinical results. Surg Gynec Obstet $1924 ; 39: 701-20$.

Southwick WO, Robinson RA. Surgical approaches to the vertebral bodies in the cervical and lumbar regions. J Bone Joint Surg [ Am] 1957; 39-A :631-43.

Wong K, Waters CM, Walesby RK. Haemoptysis after Dwyer instrumentation for idiopathic scoliosis. $J R$ Soc Med 1991; 84 : 685-6. 